\title{
Functional Approach for Summative Risk Management of Medical Products
}

\author{
Roberto RIASCOS ${ }^{\mathrm{a} 1}$, Tomislava MAJIĆ ${ }^{\mathrm{bc}}$, Egon OSTROSI ${ }^{\mathrm{d}}$, Jean-Claude SAGOT ${ }^{\mathrm{d}}$ \\ and Josip STJEPANDIĆ \\ ${ }^{a}$ Roche Diabetes Care GmbH, Germany \\ ${ }^{b}$ University North, Koprivnica, Croatia \\ ${ }^{c}$ Croatian Academy of Sciences and Arts in Diaspora and Homeland (HAZUDD), St. \\ Gallen, Switzerland \\ ${ }^{d}$ ERCOS/ELLIADD EA4661, Univ. Bourgogne Franche-Comté, UTBM, F-90010 \\ Belfort, France \\ ePROSTEP AG, Germany
}

\begin{abstract}
In the search to maintain the integrity of all systems and components over the span of the product's life, a tightly integrated risk management process and system shall enable a discrete but frequent enough calculation method. The challenge is finding a methodology where risk management is living, concomitant process throughout the lifecycle, which is cumulative, so the risk assessments refine with the product's definition while adhering to a tightly regulated enviroment for medical devices. This process needs be fully embedded in the PLM processes so it is able to accompany the product at any change throughout its life. The Multiinterfaces Entity Model (MIEM) was introduced integrated with a summative risk management concept that allows an incremental risk analysis, while the entities in the MIEM are refined. The results were a recursive methodology that supported with a PLM integrated risk management, can be automated to make sure the risk assessment is complete, re-usable and configurable as an additional PLM function. The focus of this paper lies on the generation of the functional structure of a product which is connected with the Multi-interfaces Entity Model. This allows to create a configured design structure, applying recursively the same cumulative properties of the risk assessment where a new configuration would create new interfaces.
\end{abstract}

Keywords. Product Risk Management, Configuration Management, Modular Design, Product Lifecycle Management

\section{Introduction}

Modern medical devices are launched with ever higher expectations with regard to functional requirements and shorter development times due to market pressure. This development implies new working approaches including both supporting methods and tools, especially if the supervisory authorities monitor and validate the product's compliance. Medical Devices include instruments, apparatus and software intended for use in diagnosis, cure or prevention of diseases in humans or other animals [25]. Product development of highly regulated products as known in medical industry is usually facilitated by contemporary PLM systems as an information backbone that reflects and

${ }^{1}$ Corresponding Author, Mail: roberto.riascos@roche.com. 
supports various business processes. With this system changes can be approached in a systematic manner and their effects are continuously provided transparent by the configuration management [1].

The continuous quality enhancement is one of the dominating approaches in the industrial process improvement [2]. Attempting to maintain the desired integrity of all systems and components over the span of the product's life, a tightly integrated product risk management process and system shall enable a discrete but frequent enough method to assess potential hazards, and to evaluate whether changes are necessary or if the next step of in the product's lifecycle can be started [3]. The challenge is finding a methodology where risk management is living process throughout the lifecycle, that is (a) cumulative so the risk assessments refine with the product's definition from the first idea on and the sum of the component risks add up to the entire product's risk assessment, and (b) embedded in the PLM processes so it is able to accompany the product throughout its life representing all variants and changes. It shall comprise all activities, from the technology roadmap to the start of production, including common and approved methods; e.g. Failure Mode and Effect Analysis (FMEA), Value Analysis, Kano model [4].

The outline of the paper is as follows. In section 1, the background of risk management and PLM is introduced. In section 2, the problem statement is presented. The solution concept is explained in section 3. Discussion of the challenges that still exist in the manufacturing domain is presented in section 4 . The paper ends with a brief conclusion and outlook.

\section{Background}

Risk is a natural companion of all activities of the company, at all levels, and can result in visible deterioration in the quality of products and services, and, therefore, a decline in competitiveness [5]. Therefore, risk is often subject of internal and external regulation and audits (e.g. by a regulation body) to ensure devices meet user's needs and intended use. The business environment described in this paper, in which the global medical device manufacturer operates, is strictly regulated [6]. Just as regulators have increased their ability to receive information electronically, in terms of the speed and growing capacity to analyze large amounts of data, so do medical device manufacturers, in order to anticipate and detect risks resulting from product failure or poor performance. In this sense, they should have higher reaction rates than regulators [7].

Unlike risk, which is defined as a known but potentially unfavourable outcome, the probability of which can be estimated, called uncertainty, is a situation where a decision has more than one possible outcome and in which the probability of adverse outcomes is unknown, due to lack of information or unstable variable structure in the given environment [10].

Risk management involves the use of consistent and repeatable procedures with the aim of identifying, assessing and interpreting risk in the given scenarios so that risky activities could be monitored [11] and controlled. Risk management does not seek to eliminate risks - it is unable to do so, but it creates an environment in which optimal business decisions can be made taking into account identified risks, developing potential risk management alternatives and identifying their associated costs and benefits [12]. The technical risks associated with the product primarily are based on insufficient knowledge and experience with the used technology, and may have consequences for 
quality, costs and deadlines. They often arise due to the budgetary constraints during the development project [13].

Once the risks have been identified and gone through the above procedures, it is crucial to select an appropriate technique that will effectively eliminate or control the risk exposure. There are four potential responses to risks: acceptance, avoidance, control, and transmission. When making decisions, compromises are inevitable. Trade-offs are made based on the logic that it is better to embark on a lower but known cost, in order to in turn reduce the potentially higher unknown costs associated with the risk [14].

As the main purpose of a medical device is related to the health of patients and human life, the risk management process must go through the lifecycle of the medical product in order to avoid potential dangers. Product quality metrics in the future will go in the direction of data enrichment in order to get a clearer picture of product quality risks [6].

Addressing the cumulative risk assessments, it is an opportunity to create an appropriate product risk model with interdependencies between product components. With this, the usage of the Multi-interfaces Entity Model (MIEM) can be investigated in order to define the interdependencies between components and functions that will enable a risk management concept that is incremental and adjusted with product refinement to be in line with re-usable assets, modularity and configuration of products. During design milestones, when a certain degree of product maturity is achieved, the risk assessment of the product shall be crucial input to confirming the gate for the next step in development.

There are a number of risk evaluation methods available focused on different goals; e.g. project management, products, organization [8]. However, the purpose of this paper is to explore an implementable model for risk handling in a more precise and integrated manner rather than to develop a new method. This enables a holistic product risk assessment based on reused components, systems and modules of the product as family of variants. The outcome would yield a recursive methodology that supported with a PLM integrated risk management, can be automated to make sure the risk assessment is complete, reusable and configurable [14]. If this concept would be implemented with the PLM architecture, it would provide to support further views of the products for the nest lifecycles: production planning, production, assembly, operation, maintenance and recycling [15].

\section{Problem statement}

The goal of an effective product risk management system is to allow continuous business operation and achieve the goals of an organization. In order to achieve this product risk management should be a living actor of the design process, linked to regulatory compliance needs to roll-out a product while meeting market and customer requirements. Integrating risk management to the design process enforcing it as real-time development driver ensures better design and project decisions, shortening time-to-market and reducing the risk of cost intensive product failures during the phases of usage and maintenance. As risk management is a process that accompanies the product during these later life-cycle phases it becomes also a driver of better new product development.

The sustained success of technical risk management is possible only through the efficient control of risks based on singular items or systems of a product. Different methods (FMEA, DRBFM etc) need to be applied for any instance of a product or product families, for this reason risk management needs to be used within the context of 
reusables assets, browsing through the product structure and considering all configured items. Therefore, the risk assessment and control methods need to become fully interoperable with configuration management within PLM as a fully integrated process, method and application [16]. As basis for the method, leveraging the structured nature of PLM product representation, the items in the product structure can be associated to a vector of failures, evaluations and controls, enabling a systematic identification of risks based on the aggregation of the singular items and their associated risk vector. The goal is the transparent presentation of all risk consequences, which then will be assessed qualitatively and quantitatively based on a specific product configuration [17]. The impact on each change of an item and the associated risk assessment of the singular parts can be directly identified being able to extrapolate the impact in case that the items is reused in several product configurations.

With an appropriate model and method, the elimination of deficits (time delay, lower accuracy, etc.) within the assessment of interdependencies in risk consequences and the efficient control of technical risks becomes possible. The assessment and control of technical risks for any configuration need to complete the closed loop of product risk management within PLM that contains the process phases identification, analysis and assessment and control. In such a way, the overall gain of knowledge in form of verification and validation of product risk assessments can be realized. The impact of any design change can be investigated in the early phase of the product development. The simulation of risk control measures should ensure the success of product risk management [18]. The developed conceptual solution should fulfil the requirements for product development of medical products based on international standards (FDA, ISO14971).

Finally, the formalisms for product assessment of medical devices should be proposed. To make these formalisms fully operational, several research issues should be addressed [19]. What are the relevant characteristics of a configurated system based product with regard to product risk management [20]? How these characteristics can be described in a formal way to propose appropriate methods? How the risk vectors can be mapped to product structure components and modules and used subsequently in similar products [21]?

\section{Conceptual solution}

This conceptual solution aims is to create risk assessments in each step of the development process understanding the data describing the product will evovle with each lifecycle, fom the product ideation to the design phase, throughout the saturation lifecycle (operation, maintenance, etc.) until the end-of-life. Levering the PLM Systems ability to create filtered views to make the product data available for different processes and needs of business. The foundation of creating views in PLM are a product strucuture [15]. The product structure evolves steadily while the product matures. Specific configurations such as E-BoM, M-BoM, as designed, as ordered, as serviced, etc. represent different views for different purposes.

The concept proposed in this paper is based on the asumpution we are updating an existing product and that the performance information is accesible, and appropriate requirements that ensure user needs and intended use are documented in a measurable way. The idea is that with this information, designers can start decomposing the product using the Multi-interface Entity Model (MIEM) [22]. This modelling technique is 
performed iteritably until a complete description of the entities and interfaces that compose the product is well defined. The MEIM methodology considers the products as entities and their directly associated interfaces in a top-down manner. At first the product is seen a black box, one entity, plus its external interfaces. Subsequently every entity is then decomponsed into sub-entities, resulting in new interfaces between these as shown in Figure 1.
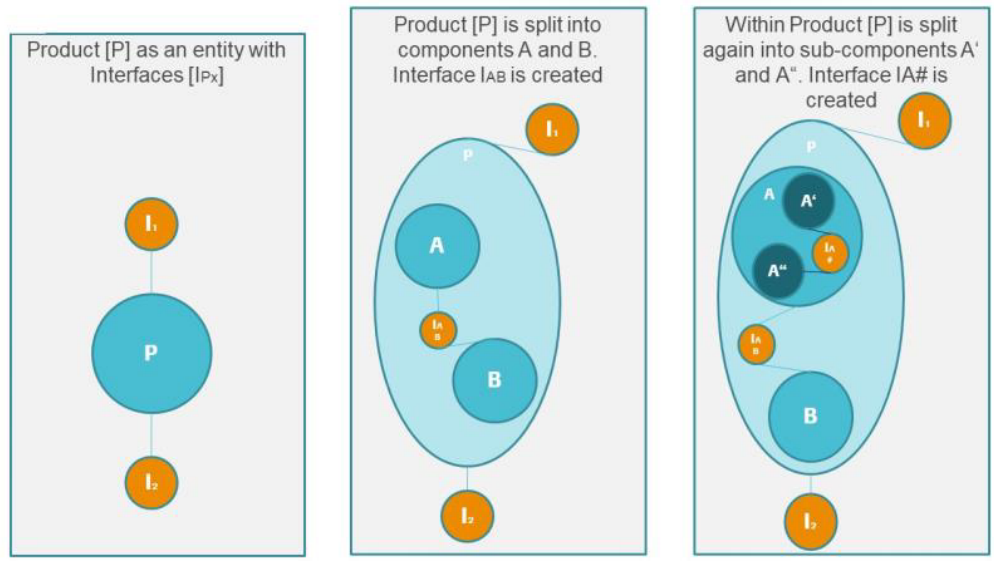

Figure 1. Decomposition of a Product using the Mult-interfaces Entity Model.

Figure 2 shows the MIEM methodology applied to a medical device lancet. The device of this example is the Accu-Chek ${ }^{\circledR}$ Softclix lancet which aims to safely pierce the the patient's skin to draw blood for a glucose reading in a separate device, the glocumeter. The lancet consists of a polymer body specially designed to be inserted into the Softclix Lancing Device. The lancet is fixed into the Lancing Device, the Lancing Device has a mechanisms to control the penetration deepness as well as push and retrieve mechanism to pierce the skin and quickly hide the Lancet's needle in the Lancing Device. The lancet shall not be reused but disposed of in a sharps box like other used needles. The Lancet is composed of a body that houses a needle and a cap that protects the needle and the user or patient from the needle itself.

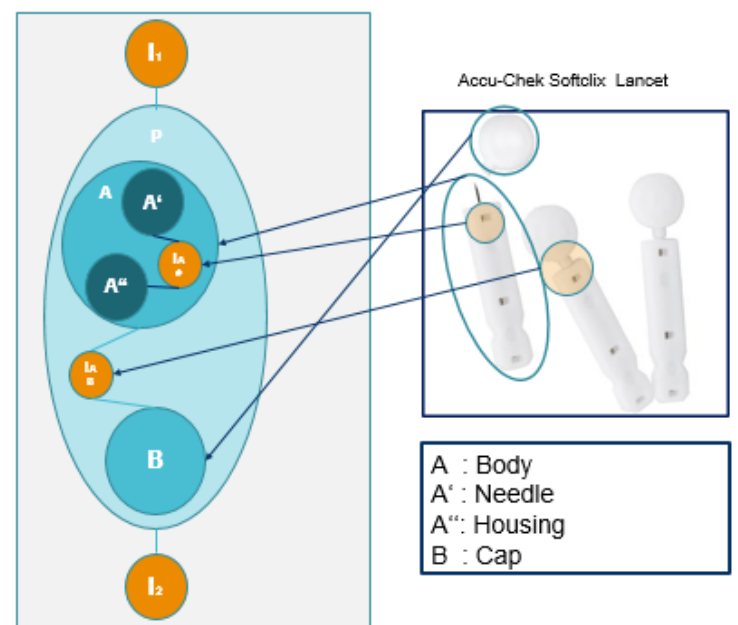

Figure 2. MIEM Applied to Accu-Chek ${ }^{\circledR}$ Softclix 
Performing the MIEM iterations delivers useful information about the product that complements the product requirements and needs. This information is used to create a functional achitecture that lists the expected functionalities of the product, the relationships within functions and the assignements of these to an entity or interface. The functional architecture makes sure the interfaces and interactions of the product are clearly described including the import and export of infromation and energy as seen on Figure 3. Once the functional architecture is built, a risk assessment can be performed and the risks assigned to the individual entities and interfaces of the model. The risk values are derived from the known information from the product requirements as a function of the relationships defined in the functional architecture. This architecure sets the stage for a collection of risks assessemnts at the level of functions independent of the future proposed solutions that can be used in a cumulative manner to produce the risk assessemnt for a specific product [23] in a later stage of the development process. The details on how the MIEM was applied to the Accu Chek ${ }^{\circledR}$ Softclix device, the definition entities and interfaces, their raltionship, how a functional architecture was constructed out the MIEM and the information gained from it, are throughtly described in a previous paper [22]

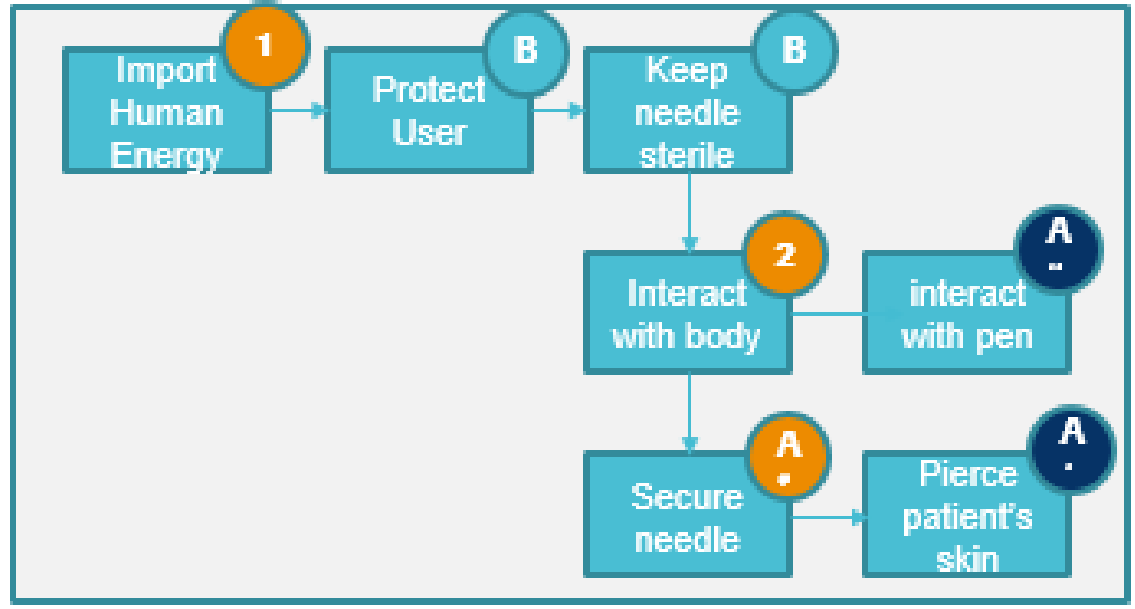

Figure 3 Creating a functional architecture of the product and assigning the functions to the MIEM entities

Keeping in mind the goal to reflect this information in a PLM environment the MIEM and functional architecture deliver the first steps to organize data in structures and concatenate information and create meaningful traces of information. At this point the designers have the possibility to create the Defintion View in PLM tracing requirements, functions, entities and interfaces, and risk vectors that include the risk description, evaluation and controls. This structure is free of any solution bias and shall give enough information if the product is viable and the next milestone of the product development can be started.

In order to reach our goal to take our existing product and have a framework to be able to define modules and re-usable assets. Modules are a set of functionalities that can have several technical solutions and can be re-used in several product configurations. To achieve this, the designers should create a logical architecture that provides a detailed description of the modules without defining the module's technology but clearly defining to which entities or interfaces of the MIEM it belongs as well as the inputs and outputs 
for, for example, energy or information. Having this view of the product allows the risk managers to create a new level of the product risk analysis. Being that a module is the sum of several functions, the risk assessment of the module is the cumulation of the risks associated to each function plus the risk analysis of the module itself as a function of clustering done for the module.

With well defined modules, designers can start proposing solutions that fulfill the modules in a layer called the generative architecture with the end goal to describe the set of all components of a logical architecture and defines the technology of each component. For each defined module, there could be several solutions. This creates a network of possible combinations of solutions where all possible solutions of a module could theoritically be matched with all solutions of a further module linked in the logical architecure. Some of these combinations are incompatible and should be promptly ruled out. For the remaining possible combinations, the designers have now the possibility to create a risk assessment for each pairing. The risk vector at this point is not associated to the module A or to the related module B but to the link between modules A and B. This exercise will give the designers the chance to create a layer of physical products in the physical architecture. The possible products are a product of the possible combinations between modules given they are compatible. At this point the cumulative risk assessment across the architectures shall give the designers which module combination describes the product with the lowest risk (Figure 4).

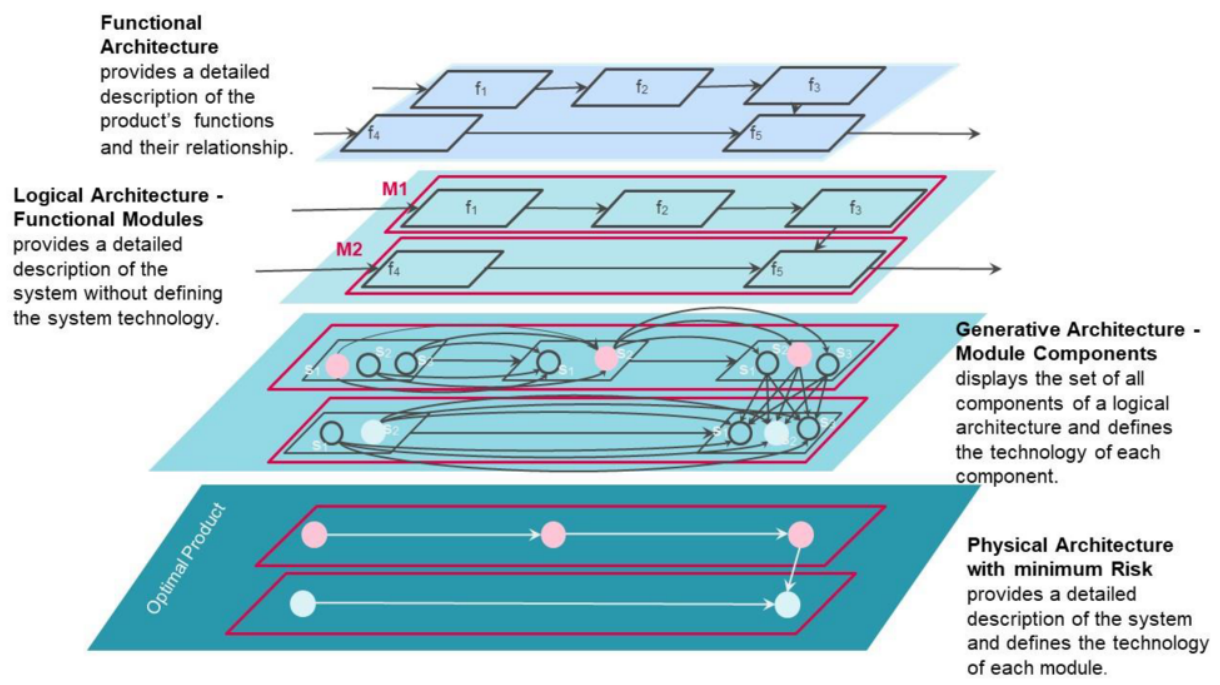

Figure 4 Multi-layered Architecture Approach

Just as the different layers of architecture deliver the traceability from the requirement to the function to the module to the products, the information can be reflected in PLM structures using views that serve for orientation and traceability of the content. Out of the functional architecture an as defined structure of the product can be created. The logical architecture gives an overview of which configuration items are needed in the design structure and the generative architecture can be represented as $150 \%$ product structure where viable configurations can be destiled through the variant 
configuration management process as seen on Figure 5. Here, the logical architecture provides the information of the configuration items (CI) that the as-designed product structure will have. The CIs will then be the structural parent of the possible solutions to these modules.

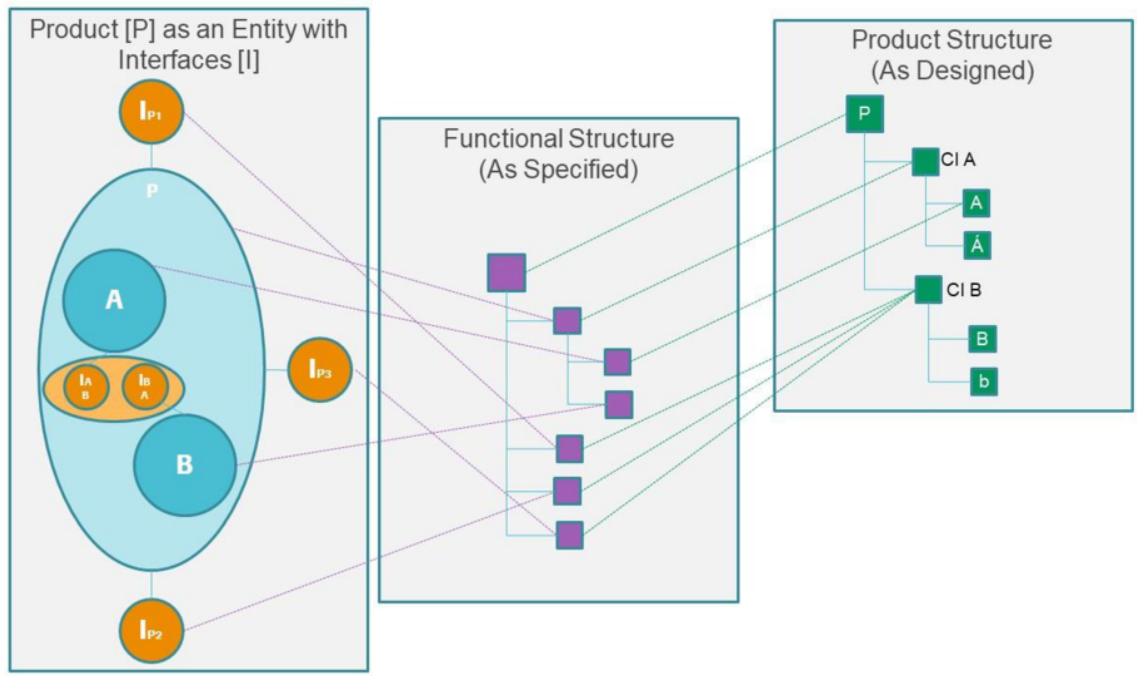

Figure 5 Creating PLM Views as an Output of the MIEM and the Derived Architectures

\section{Discussion}

In this paper, a conceptual solution for the integration of product risk management in a comprehensive PLM approach for highly configured, modular medical devices is explored. In this way, the standard PLM system is being enhanced by an additional capability as an intelligent collaborative and distributed platform for assessment, maintenance and monitoring of each product capability during the product lifecycle. Thus, the proposed, generic solution has the potential to fulfil the demand of the continuous, software-aided risk assessment and to provide the appropriate user-friendly support within PLM system. Finally, the resulting risk management process looks as seamless as possible, with the purpose to provide a basis for the software solution to achieve the desired goal: identifying, assessing, prioritizing, or monitoring risk like other summative properties (e.g. the cumulative weight of the configured product) are examined in an assembly.

Methods implemented by software solutions simplify the daily work of risk engineers and managers that all risks which are accompanied with a product configuration are properly considered and minimize the subjectivity of subsequent human risk assessments. Although the risk values do not provide the absolute results, their comparison is a valuable basis for reproducible risk assessment. This generic methodology can be used for all types of product risk assessments, not dependent on the domain, the type and the genesis of the risk. Risk assessment emerges while the product emerges during its product lifecycle. The uncertainty remains only through little human assistance and judgment. However, the comparison stabilizes this activity too. The 
proposed solution can also facilitate that the effects of cognitive biases are documented within the risk matrix.

The approach to map the function structure in the design architecture is just the first step, followed ba a comprehensive definition of the risk model. In that way, a methodology can be defined which is valid for the entire product lifecycle. Assumed a performant software implementation, the method monitors the potential technical risks in a systematic manner at each moment in the product lifecycle. In particular, a standardized risk assessment is beneficial in case of product change.

\section{Conclusions and outlook}

Focusing cumulative risk management methodology, we investigated the usage of the Multi-interfaces Entity Model (MIEM) integrated with a summative risk management concept that allows an incremental risk analysis, as the entities in the MIEM are refined. After a certain degree of maturity, the risk assessment of the product a design architecture of the product is generated where the risk analysis validated confirming the gate for the next step in development. Within the design structure the same principle is applied the sum of the risks of the component and the interfaces will define the risk assessment of the product. This approach does risk management not only at the global (top down) or component (button up) level but at the modular level. The modular approach can be scaled up to large component clusters down to design elements and features of the PDM system at any level where re-use is possible. Perfomring a risk assessment at the functional level provides a new dimension of risk management of components or modules as a parmeter of the function they provide. Further from this paper, if this assessment is validated the next step is to create a configured design structure, applying recursively the same cumulative properties of the risk assessment where a new configuration would create new interfaces. The configured structure describes the product in the design view and should create the next level of traceability from the requirement to the function over to the solution. This enables a holistic risk assessment based on reused components, systems and modules of the product as family of variants. The intention is to create further views of the product to asess the risk of later life-cycles also at the the module level but as a function of their manufacturability, commerciability, recycle-ability amoing others. This, supported with a PLM integrated risk management as described in previous papers, can be automated to make sure the risk assessment is complete, re-usable and configurable.

The next steps are the implementation in this concept with the PLM architecture and make it possible to support further views of the products for the nest lifecycles: production planning, production, assembly, usage, maintenance and recycling. With this approach the intention is to avoid having a very heavy risk management at the definition and design phase that is later left to a lower plane in the life of the product.

In this way, the product risk assessment can be established a an inherently transdisciplinary engineering process[24].

\section{References}

[1] J. Stark, Product Lifecycle Management (Volume 4): The Case Studies, Springer Nature Switzerland AG, 2019. 
[2] S. Manz, Medical Device Quality Management Systems. Strategy and Techniques for Improving Efficiency and Effectiveness, Academic Press, London, 2019.

[3] M. Leimeister and A. Kolios, A. A review of reliability-based methods for risk analysis and their application in the offshore wind industry, Renewable and Sustainable Energy Reviews, 2018, Vol. 91, 1065-1076.

[4] X. Zhao and X. Bai, The Application of FMEA method in the risk management of medical device during the lifecycle, 2010 2nd International Conference on E-business and Information System Security, 22-23 May 2010, DOI: 10.1109/EBISS.2010.5473713, pp. 455-458.

[5] T. Aven, Foundational Issues in Risk Assessment and Risk Management, Risk Analysis, Vol. 32, No. 10, 2012, pp. 1647-1656.

[6] J. Hatcliff, E.Y. Vasserman, T. Carpenter and R. Whillock, Challenges of distributed risk management for medical application platforms, 2018 IEEE Symposium on Product Compliance Engineering (ISPCE), 1416 May 2018, DOI: 10.1109/ISPCE.2018.8379270.

[7] D.C. Hall, Making Risk Assessments More Comparable and Repeatable, Systems Engineering, Vol. 14, No. 2, 2011, pp. 173-179, DOI 10.1002/sys.20169.

[8] B. Pongrac, T. Majić, Business Risk Management, Technical Journal, Vol. 9, 2015, No. 1, pp. 94-98.

[9] M.N. Teferra, ISO 14971 - Medical Device Risk Management Standard, International Journal of Latest Research in Engineering and Technology (IJLRET), 2017, Vol. 3, Issue 3, March 2017, pp. 83-87.

[10] T. Aven and E. Zio, Foundational Issues in Risk Assessment and Risk Management, Risk Analysis, Vol. 34, No. 7, 2014, pp. 1164-1172.

[11] J. Oehmen, B. Dick, U. Lindemann and W. Seering, Risk Management in Product Development - Current Methods, International Design Conference - DESIGN 2006 Dubrovnik - Croatia, May 15 - 18, 2006, pp. 1551-1558.

[12] E.D. Smith, W.T. Siefert and D. Drain, Risk Matrix Input Data Biases, Systems Engineering, 2009, Vol. 12, No. 4, pp. 344-360.

[13] M.S. Kirkire, S.B. Rane and J.R. Jadhav, Risk management in medical product development process using traditional FMEA and fuzzy linguistic approach: a case study, Journal of Industrial Engineering International, 2015, Vol. 11, pp. 595-611.

[14] R. Riascos, T. Majić, E.Ostrosi and J. Stjepandić, Modular Approach to Technical Risk Management in Product Lifecycle Management, Advances in Transdisciplinary Engineering, 2019, Vol. 10, pp. 380-389.

[15] J. Stark, Product Lifecycle Management (Volume 2): The Devil is in the Details, 3rd edition, Springer International Publishing AG, 2016.

[16] M. Rusu, The configuration management requirements for aviation, space and defense organizations, INCAS Bulletin, 2019, 11(1), pp. 239-253.

[17] J. Stjepandić, E. Ostrosi, A.-J. Fougères, M. Kurth, Modularity and Supporting Tools and Methods, in: J. Stjepandić et al. (eds.): Concurrent Engineering in the $21^{\text {st }}$ Century: Foundations, Developments and Challenges, Springer International Publishing Cham, 2015, pp. 389-420.

[18] D. Flood, F. McCaffery, V. Casey, R. McKeever and P. Rust, A roadmap to ISO 14971 implementation, Journal of Software: Evolution and Process, 2015, Vol. 25(5), pp. 319-336.

[19] B. He, H. Xue, L. Liu, Q. Pan, W. Tang and E. Ostrosi, Rigid-flexible coupling virtual prototyping-based approach to the failure mode, effects, and criticality analysis, International Journal of Advanced Manufacturing Technology, 2019, Vol. 100(5-8), pp. 1695-1717, DOI: 10.1007/s00170-018-2641-2.

[20] J.K.Keski-Rahkonen, Probabilistic framework for product design optimization and risk management, IOP Conf. Series: Materials Science and Engineering, 2018 ,Vol. 351, 012007.

[21] B. Fontem, B. Keskin, S. Melouk, C. Vaughn, An exact decomposition algorithm for a chanceconstrained new product risk model, Operations Research Letters, 2019, Vol. 47, Issue 4, pp. 250-256.

[22] R.A. Riascos, T. Majić, E. Ostrosi, J.-C. Sagot, j. Stjepandić, Multi-interfaces Entity Model for Summative Risk Management of Medical Products, IFIP $18^{\text {th }}$ International Conference on Product Lfiecyle Management, 11-14 July 2021, Curitiba, accepted, in press.

[23] H.P.L. Bruun, N.H. Mortensen, U. Harlou, M. Wörösch and M. Proschowsky, PLM system support for modular product development, Computers in Industry, 2015, Vol. 67, pp. 97-111.

[24] N.Wognum, C. Bil, F., Elgh, F., M. Peruzzini, J. Stjepandić, W.J.C. Verhagen, Transdisciplinary engineering research challenges, Advances in Transdisciplinary Engineering, 2018, Vol. 7, pp. 753-762.

[25] Council Directive 93/42/EEC of 14 June 1993 concerning medical devices. eur-lex.europa.eu. 